\title{
ELECTRON-MOLECULAR VIBRATION COUPLING IN TTF-BASED DONORS
}

\author{
K.I. POKHODNIA \\ Institute of Semiconductors, Ukr. Acad. of Sci., Kiev, Ukraine
}

\begin{abstract}
BEDT-TTF, BMDT-TTF and BEDO-TTF are well known thio-TTF and oxo-TTF (in the former case) derivatives on the basis of which cation-radical salts with $2 \mathrm{D}$ type of conductivity can be produced. In this work the comparative analysis of electron-molecular vibration coupling constants (obtained by MNDO quantum-chemical method) that describe the interaction between totally symmetric $A_{\mathrm{g}}$ modes and electron on HOMO level is performed.
\end{abstract}

PACS numbers: $74.70 .-b$

\section{Introduction}

It is well known that electron-phonon coupling is one of the main reasons of superconductivity (SC). In comparison with the traditional inorganic SC materials the phonon spectra of organic metals are much wider and consist of two types of vibrational modes: intermolecular acoustic phonons and intramolecular vibrations of atoms or atomic groups of organic molecules. It is rather difficult to explain the high value of $T_{\mathrm{c}} \approx 12 \mathrm{~K}$ in some of BEDT-TTF (bis(etylenedithio)tetrathiafulvalene) [1] salts only by interaction with the phonons of the first type. Intermolecular vibrations could be a significant source of phonon exchange for SC pairing. This idea found its theoretical support in the paper of Yamaji [2]. Thus the correct evaluation of electron-molecular vibration coupling (EMVC) constants for BEDT-TTF and relative donors is of great importance.

\section{Results and discussion}

There are two main methods of the estimation of EMVC constants. First, the so-called dimer method based on fitting the model reflectivity spectrum (where EMVC constants $g_{i}$ are model parameters) to the experimental one, which contains the strong vibronic features due to EMVC [3]. The second one is based on the direct calculation of the derivative $\left(\partial \varepsilon_{\gamma} / \partial Q_{i}\right)$ (where $\varepsilon_{\gamma}$ is the energy of highest occupied molecular orbital (HOMO) level calculated by MNDO quantum-chemical 
method and $Q_{i}$ is a normal coordinate) which is proportional to $g_{i}$ [4]. The principal limitation of the first model is the fact that it was designed for the array of insulating dimers. The monocrystals with such structure are not available for BEDT-TTF family substances. The attempt to use this model for describing the reflectivity of $\beta$-BEDT-TTF ${ }_{2} \mathrm{I}_{3}$ conductive salt gave obviously understated $g_{i}$ values $\sum_{i} g_{i} N(0)<0.1[5]$.

The second method is more universal but it takes a lot of spectroscopic work. Taking into consideration the specific fea iures of this method, principal attention was focused on proving the adequacy of a chosen valence force field (VFF) for the description of molecular vibration in this class of donors. The variant of VFF proposed in our earlier work for BEDT-TTF and its deuteroanalogue [6] was easily adopted for substituted terminal groups BMDT-TTF (bis(methylenedithio)TTF) [7] including the case of its cation radicals [9] and for $\mathrm{S} \rightarrow \mathrm{O}$ substituted BEDO-TTF [8].

Thus, it could be concluded that the chosen VFF adequately describes the vibration in this TTF derivatives. By performing the normal coordinate analysis the displacements of each atom for all modes allowed to couple by symmetry were obtained and after normalization they were used for EMVC constants calculation.

In Table the EMVC constants for all $A_{\mathrm{g}}$ modes of TTF, BMDT-TTF, BEDT-TTF and its cation-radicals together with BEDT-TTF are presented. For TTF and its thio-derivatives the highest values of EMVC constants have modes $\omega_{2}, \omega_{3}$ (central and ring $\mathrm{C}=\mathrm{C}$ double bonds stretchings) and modes $\omega_{6}$ for TTF, $\omega_{7}$ for BMDT-TTF, and $\omega_{9}, \omega_{10}$ for BEDT-TTF (C-S bond stretchings in central and peripheral heterocycles). Orbitals of these atoms give the main input in HOMO and their motion causes the significant shift of HOMO energy. In cation-radicals only in-phase $\mathrm{C}=\mathrm{C}$ stretching mode $\omega_{3}$ and $\mathrm{C}-\mathrm{S}$ modes $\omega_{6}, \omega_{7}, \omega_{9}$ (for TTF ${ }^{+}$, $\mathrm{BMDT}^{-\mathrm{TTF}^{+}}$and BEDT-TTF ${ }^{+}$respectively) have the biggest $g_{i}$ values due to the strong form mixing.

In the case of BEDO-TTF the introduction of strongly electronegative oxygen atoms leads to sufficient $\pi$-electron density shifts to the peripheral cycles, though the biggest its part retains on central TTF fragment. But EMVC constants of $\mathrm{C}=\mathrm{C}$ and $\mathrm{C}-\mathrm{S}$ stretching modes $\omega_{2}, \omega_{3}, \omega_{9}, \omega_{11}$ are still the highest and $\mathrm{C}-\mathrm{O}$ and $\mathrm{C}-\mathrm{C}$ stretching modes $\omega_{6}, \omega_{7}, \omega_{8}$ also have the comparable values of $g_{i}$. Corresponding vibronic bands were observed in IR spectra of BEDO-TTF compounds [8].

One can see that above mentioned EMVC constants for BMDT-TTF and BEDO-TTF are even higher than those for BEDT-TTF. It might be presumed that the search for $\mathrm{SC}$ on their basis is still promising.

Thus the fundamental data obtained allow to perform quantitative theoretical analysis of EMVC input in SC pairing and give grounds for interpretation of the series of intensive bands in IR spectra of the conductive compounds of analyzed donors. 
TABLE EMV-constants for $A_{\mathrm{g}}$ modes of TTF, BEDT-TTF, BMDT-TTF and BEDO-TTF (a) and their cation-radicals (b).

\begin{tabular}{|c|c|c|c|c|c|c|c|c|}
\hline \multicolumn{9}{|c|}{ (a) } \\
\hline \multirow[b]{2}{*}{$i$} & \multicolumn{2}{|c|}{ TTF } & \multicolumn{2}{|c|}{ BEDT-TTF } & \multicolumn{2}{|c|}{ BMDT-TTF } & \multicolumn{2}{|c|}{ BEDO-TTF } \\
\hline & $\begin{array}{c}\omega_{i} \\
{\left[\mathrm{~cm}^{-1}\right]}\end{array}$ & $g_{i}$ & $\begin{array}{c}\omega_{i} \\
{\left[\mathrm{~cm}^{-1}\right]}\end{array}$ & $g_{i}$ & $\begin{array}{c}\omega_{i} \\
{\left[\mathrm{~cm}^{-1}\right]}\end{array}$ & $g_{i}$ & $\begin{array}{c}\omega_{i} \\
{\left[\mathrm{~cm}^{-1}\right]}\end{array}$ & $g_{i}$ \\
\hline 1 & 3076 & 0.028 & 2932 & 0.011 & 2948 & 0.006 & 2954 & 0.027 \\
\hline 2 & 1554 & 0.675 & 1556 & 0.297 & 1553 & 0.374 & 1654 & 0.425 \\
\hline 3 & 1500 & 0.697 & 1509 & 0.566 & 1483 & 0.595 & 1527 & 0.549 \\
\hline 4 & 1079 & 0.124 & 1394 & 0.029 & 1414 & 0.054 & 1454 & 0.045 \\
\hline 5 & 737 & 0.172 & 843 & 0.012 & 1256 & 0.076 & 1279 & 0.092 \\
\hline 6 & 463 & 0.560 & 685 & 0.163 & 982 & 0.014 & 1203 & 0.241 \\
\hline 7 & 235 & 0.120 & 508 & 0.336 & 900 & 0.030 & 1014 & 0.153 \\
\hline 8 & & & 470 & 0.027 & 656 & 0.156 & 860 & 0.300 \\
\hline 9 & & & 381 & 0.226 & 488 & 0.506 & 423 & 0.555 \\
\hline 10 & & & 146 & 0.316 & 440 & 0.341 & 590 & 0.153 \\
\hline 11 & & & & & 302 & 0.035 & 474 & 0.463 \\
\hline 12 & & & & & 146 & 0.179 & 173 & 0.115 \\
\hline$\sum^{*}$ & 20 & & $\overline{90}$ & & 11 & & 15 & \\
\hline
\end{tabular}

(b)

\begin{tabular}{|c|c|c|c|c|c|c|}
\hline \multirow[b]{2}{*}{$i$} & \multicolumn{2}{|c|}{ TTF $^{+}$} & \multicolumn{2}{|c|}{ BEDT-TTF $^{+}$} & \multicolumn{2}{|c|}{ BMDT-TTF $^{+}$} \\
\hline & $\begin{array}{c}\omega_{i} \\
{\left[\mathrm{~cm}^{-1}\right]}\end{array}$ & $g_{i}$ & $\begin{array}{c}\omega_{i} \\
{\left[\mathrm{~cm}^{-1}\right]}\end{array}$ & $g_{i}$ & $\begin{array}{c}\omega_{i} \\
{\left[\mathrm{~cm}^{-1}\right]}\end{array}$ & $g_{i}$ \\
\hline$\overline{1}$ & 3078 & 0.074 & 2896 & 0.032 & 2912 & 0.022 \\
\hline 2 & 1496 & 0.054 & 1461 & 0.171 & 1465 & 0.165 \\
\hline 3 & 1418 & 0.869 & 1419 & 0.765 & 1427 & 0.746 \\
\hline 4 & 1074 & 0.104 & 1380 & 0.003 & 1421 & 0.102 \\
\hline 5 & 755 & 0.077 & 854 & 0.024 & 1287 & 0.063 \\
\hline 6 & 493 & 0.795 & 668 & 0.187 & 979 & 0.140 \\
\hline 7 & 260 & 0.130 & 516 & 0.524 & 896 & 0.117 \\
\hline 8 & & & 496 & 0.098 & 672 & 0.192 \\
\hline 9 & & & 442 & 0.210 & 508 & 0.476 \\
\hline 10 & & & 174 & 0.116 & 483 & 0.050 \\
\hline 11 & & & & & 318 & 0.025 \\
\hline 12 & & & & & 159 & 0.041 \\
\hline$\sum^{*}$ & \multicolumn{2}{|c|}{178.6} & \multicolumn{2}{|c|}{132.0} & \multicolumn{2}{|c|}{127.0} \\
\hline
\end{tabular}

$\sum^{*}=\sum h \omega_{i} g_{i}^{2}(\mathrm{meV})$ is a "polaron binding energy" which equals the lowering of HOMO level resulting from its coupling with $A_{\mathrm{g}}$ modes. 


\section{Acknowledgments}

This research was partially supported by grant No. U51000 from the International Science Foundation.

\section{References}

[1] A.M. Kini, U. Geiser, H.H. Wang, K.D. Carlson, J.M. Williams, W.K. Kwok, K.G. Vandervoot, J.E. Thompson, D.L. Stupka, D. Jung, M-H. Whangto, Inorg. Chem. 29, 2555 (1990).

[2] K. Yamaji, Solid State Commun. 61, 413 (1987).

[3] M.J. Rice, Phys. Rev. Lett. 37, 36 (1976).

[4] N.O. Lipari, M.J. Rice, C.B. Duke, R. Bozio, A. Girlando, C. Pecile, Int. J. Quant. Chem. 11, 583 (1977).

[5] M.G. Kaplunov, E.B. Yagubskii, L.P. Rosenberg, Yu.G. Borodko, Phys. Status Solidi A 89, 509 (1985).

[6] M.E. Kozlov, K.I. Pokhodnia, A.A. Yurchenko, Spectrochim. Acta. A 43, 323 (1987).

[7] M.E. Kozlov, V.G. Onishchenko, K.I. Pokhodnia, Zh. Prikl. Spektrosk. 52, 622 (1990).

[8] K.I. Pokhodnia, M.E. Kozlov, V.G. Onishchenko, D. Schweitzer, J. Moldenhauer, R. Zamboni, Synth. Met. 55-57, 2364 (1993).

[9] M.E. Kozlov, K.I. Pokhodnia, A.A. Yurchenko, Spectrochim. Acta A 45, 437 (1989). 